\title{
Auxin confers protection against ER stress in Caenorhabditis elegans
}

\author{
Anupam Bhoi, Francesca Palladino and Paola Fabrizio*
}

\begin{abstract}
Auxins are plant growth regulators that influence most aspects of plant development through complex mechanisms. The development of an auxin-inducible degradation (AID) system has enabled rapid, conditional protein depletion in yeast and cultured cells. More recently, the system was successfully adapted to Caenorhabditis elegans to achieve auxin-dependent degradation of targets in all tissues and developmental stages. Whether auxin treatment alone has an impact on nematode physiology is an open question. Here we show that indole-3-acetic acid (IAA), the auxin most commonly used to trigger AID in worms, functions through the conserved IRE-1/XBP-1 branch of the Unfolded Protein Response (UPR) to promote resistance to endoplasmic reticulum (ER) stress. Because the UPR not only plays a central role in restoring ER homeostasis, but also promotes lipid biosynthesis and regulates lifespan, we suggest that extreme caution should be exercised when using the AID system to study these and related processes.
\end{abstract}

KEY WORDS: Auxin, ER stress, Unfolded Protein Response, Tunicamycin

\section{INTRODUCTION}

Auxins are a family of plant hormones that control gene expression during many processes of cell growth and development (Teale et al., 2006). Indole-3-acetic acid (IAA), the most common natural auxin in plants, is produced mainly from tryptophan, an essential amino acid for all animals. Importantly, free-living bacteria, as well as bacterial flora in the gut of animals, can also degrade tryptophan to yield indole or indole-based compounds (Bansal et al., 2010; Lee and Lee, 2010; Lee et al., 2015).

Indoles from commensal microbiota have been shown to extend the health span of diverse organisms, including Caenorhabditis elegans, Drosophila melanogaster and mice, with only a negligible effect on maximal lifespan (Sonowal et al., 2017). In worms and flies, the effects of indoles on health span depend upon the aryl hydrocarbon receptor (AhR; also known as dioxin receptor). In C. elegans, transcriptional analysis showed that indole and its derivatives are important signalling molecules during bacterianematode interactions (Lee et al., 2017).

Laboratory of Biology and Modelling of the Cell, Ecole Normale Supérieure de Lyon, CNRS UMR5239, INSERM U1210, Université de Lyon, 69007 Lyon, France.

*Author for correspondence ( paola.fabrizio@ens-lyon.fr)

(iD) P.F., 0000-0002-1144-7727

This is an Open Access article distributed under the terms of the Creative Commons Attribution License (https://creativecommons.org/licenses/by/4.0), which permits unrestricted use, distribution and reproduction in any medium provided that the original work is properly attributed.

Received 17 November 2020; Accepted 22 December 2020
Whether treatment of C. elegans with exogenous indoles has an impact on animal physiology has not been explored. This has important implications when using the recently developed auxininducible degradation system (AID) that depends on the ability of IAA and other auxins to bind to the F-box transport inhibitor response 1 (TIR1) protein. TIR1 is the Arabidopsis-specific substrate-recognition component of the conserved SKP1-CUL1-Fbox (SCF) E3 ubiquitin ligase complex, and carries out its function only in the presence of auxin. Once bound to auxin, exogenous TIR1 targets AID-tagged proteins for ubiquitin-dependent proteasomal degradation, allowing for highly efficient, conditional protein depletion in many systems (Camlin and Evans, 2019; Daniel et al., 2018; Holland et al., 2012; Lee and Lee, 2010; Natsume et al., 2016; Nishimura et al., 2009; Trost et al., 2016), including C. elegans (Martinez et al., 2020; Zhang et al., 2015).

While studying how loss of chromatin associated Heterochromatin Protein 1 affects resistance to ER stress in different tissues of $C$. elegans (Kozlowski et al., 2014), we discovered that exposure of animals to auxin significantly increases resistance to stress of the endoplasmic reticulum (ER), both throughout development and in adults. The ER constitutes the entry point into the secretory pathway and contributes to the maintenance of cellular calcium homeostasis, lipid synthesis, and transmembrane protein folding, making the maintenance of ER homeostasis an important component of animal physiology (Hetz, 2012). The accumulation of misfolded proteins in the ER by physiological, chemical, and pathological factors, activates the Unfolded Protein Response (UPR), whose role is to re-establish ER homeostasis and promote survival (Metcalf et al., 2020). We found that increased resistance to ER stress in the presence of auxin is dependent on XBP-1 and IRE-1, upstream components of the UPR, showing that auxin acts through this stress pathway to alter animal physiology. Because of the tight link between the ER stress response and essential cellular processes such as protein folding, ageing and lipid metabolism, our data suggests that auxin treatment alone may influence the outcome of experiments aimed at studying these or related processes in C. elegans, and possibly in other species.

\section{RESULTS}

Auxin mitigates ER stress induced developmental delay and lethality

Tunicamycin (TM) induces the ER stress response by inhibiting glycosylation, leading to the accumulation of unglycosylated proteins in the ER (Travers et al., 2000). In worms, treatment with TM induces severe developmental delay and lethality, with animals arresting at various stages of larval development (Shen et al., 2001, 2005). In order to assess whether the AID system can be implemented for studies of the ER stress response, we carried out control experiments in which we scored the impact of auxin (IAA) on survival of wild-type animals exposed to TM. Adult animals were transferred on plates containing $3 \mu \mathrm{g} / \mathrm{ml} \mathrm{TM}$ in the presence or 
A

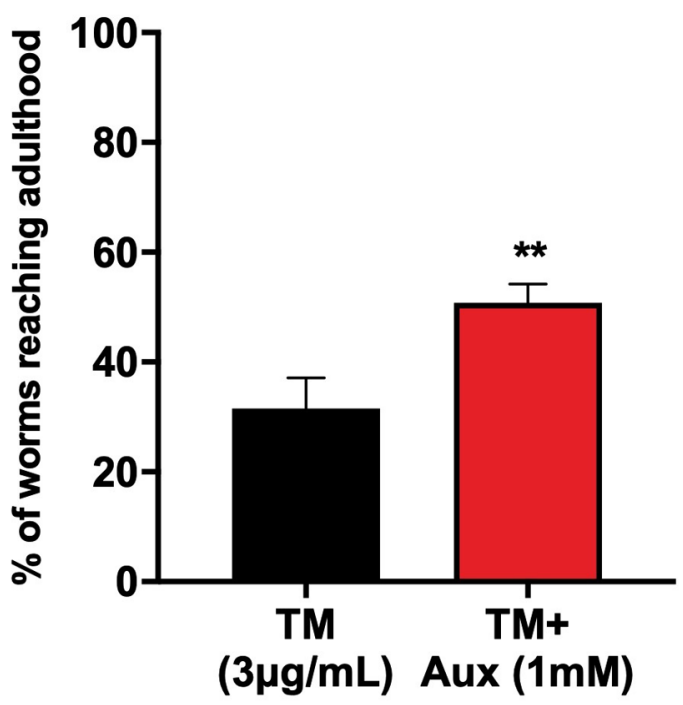

B

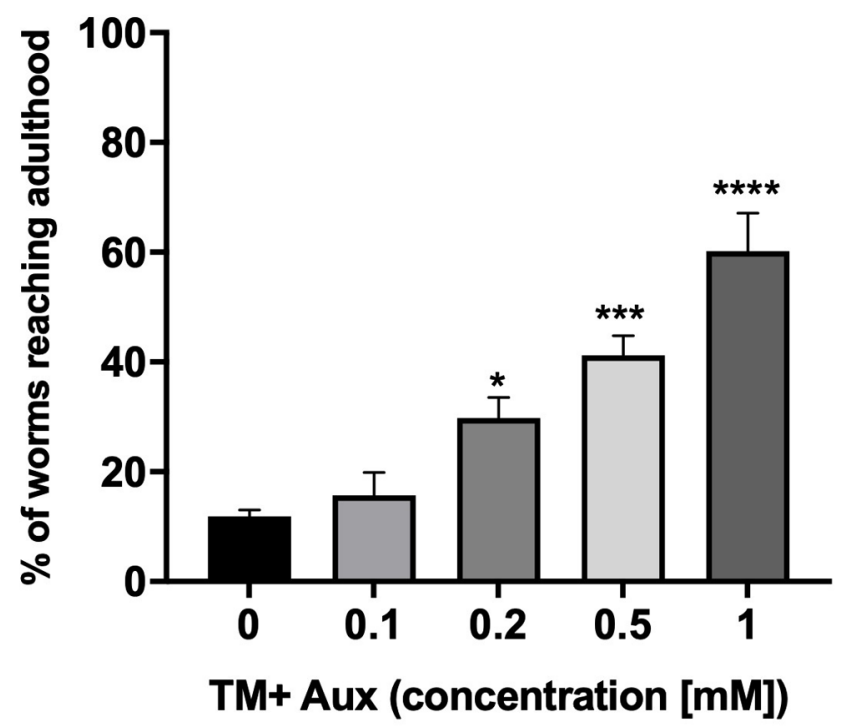

Fig. 1. Auxin protects developing worms against ER stress. (A) Proportion of wild-type animals reaching the L4-adult stage after 4 days of development on plates containing tunicamycin $(3 \mu \mathrm{g} / \mathrm{ml})$ with or without auxin $(1 \mathrm{mM})$. Error bars represent SEM from three independent experiments (Student's $t$-test, ${ }^{* *} P<0.01$ auxin versus no auxin). (B) Proportion of wild-type animals reaching the L4-adult stage after 4 days on plates containing tunicamycin ( $\left.3 \mu \mathrm{g} / \mathrm{ml}\right)$ combined with increasing concentrations of auxin (0.1-1 mM). Error bars show SEM from three independent experiments (one-way ANOVA with Tukey's multiple comparison test, ${ }^{\star} P<0.05,{ }^{* *} P=0.0005,{ }^{* * *} P<0.0001$ auxin versus no auxin).

absence of $1 \mathrm{mM}$ auxin and the ability of progeny to reach L4adulthood was scored after 4 days. We observed that addition of auxin significantly improved survival, with $50-60 \%$ of animals developing to adulthood compared to $10-30 \%$ on TM alone (Figs 1 and $3 \mathrm{~B})$. Dose response experiments with auxin concentrations ranging from 0.1 to $1 \mathrm{mM}$ in the presence of $3 \mu \mathrm{g} / \mathrm{ml} \mathrm{TM}$ revealed increased survival with increasing auxin concentrations (Fig. 1B). These results show that auxin protects animals from ER stress.

\section{Auxin also shows a protective effect in adult animals}

To test whether auxin exerts its effect uniquely throughout development, or also in mature adults, we performed survival assays on day 1-adult worms transferred on plates containing $40 \mu \mathrm{g} / \mathrm{ml} \mathrm{TM}$ in the presence or absence of $1 \mathrm{mM}$ auxin. A modest but significant $10 \%$ mean survival extension was observed in the presence of auxin, indicating that the protective effect of auxin against ER stress is not limited to developmental stages (Fig. 2; Fig. S1).

\section{Auxin increases resistance to ER stress through the canonical UPR pathway}

Disruption of ER function due to ER stress activates the UPR, which re-establishes ER homeostasis and promote survival via the upregulation of ER chaperones and components of ER-associated

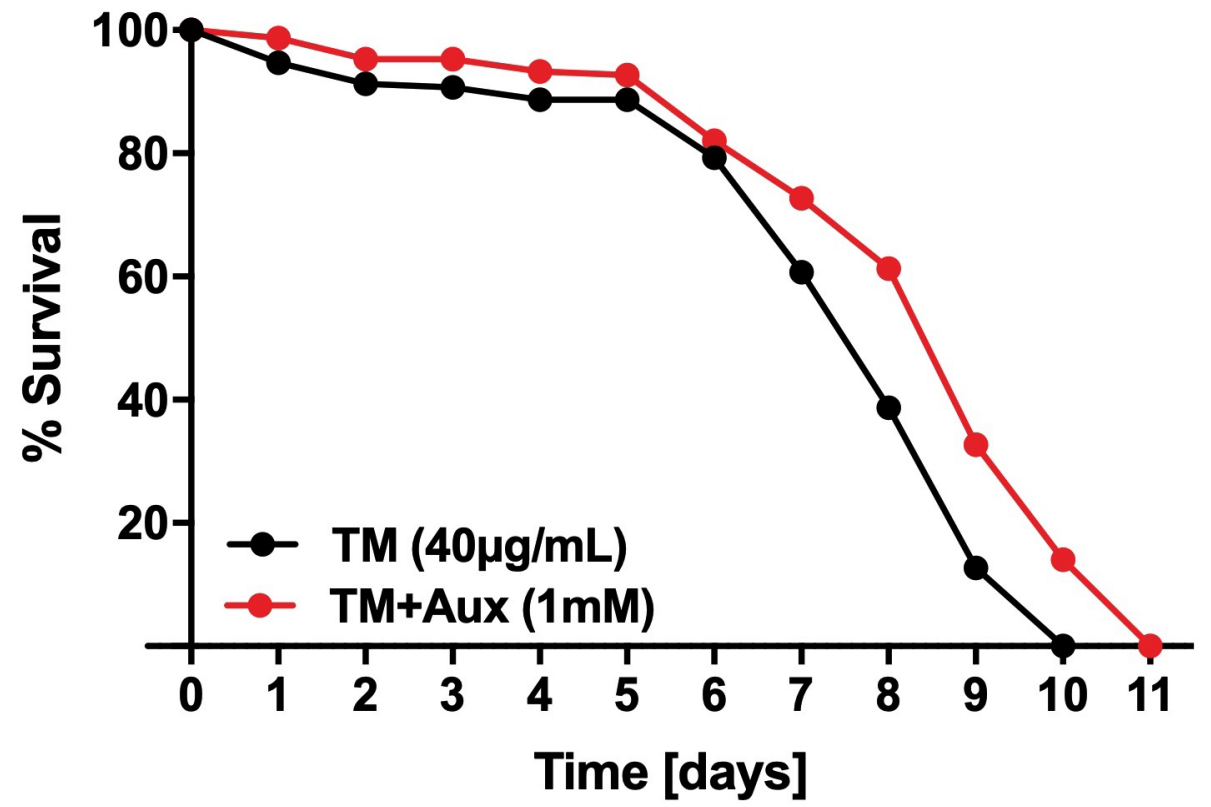

Fig. 2. Auxin promotes ER stress resistance in adult worms. Survival of adult wild-type animals on plates containing either tunicamycin $(40 \mu \mathrm{g} / \mathrm{ml})$ alone $(N=147)$, or tunicamycin and auxin $(1 \mathrm{mM})(N=144) .(P<0.0001)$. Worms were exposed to tunicamycin with or without auxin starting from the first day of adulthood (day 0 ). The $P$-value was calculated using the log-rank (Mantel-Cox) method. Mean and maximum lifespan were 7.8 and 10 , and 8.9 and 11 , for tunicamycin and tunicamycin+auxin, respectively. A replicate experiment is shown in Fig. S1. 


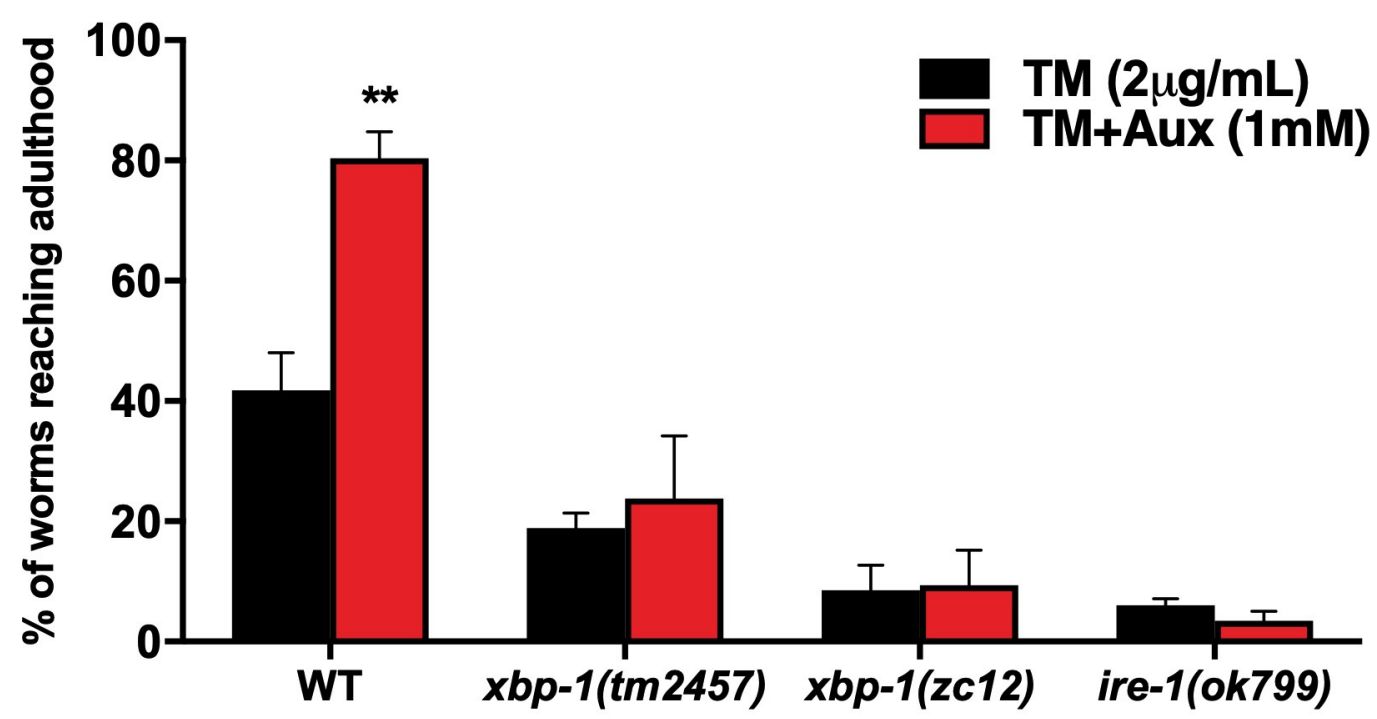

B

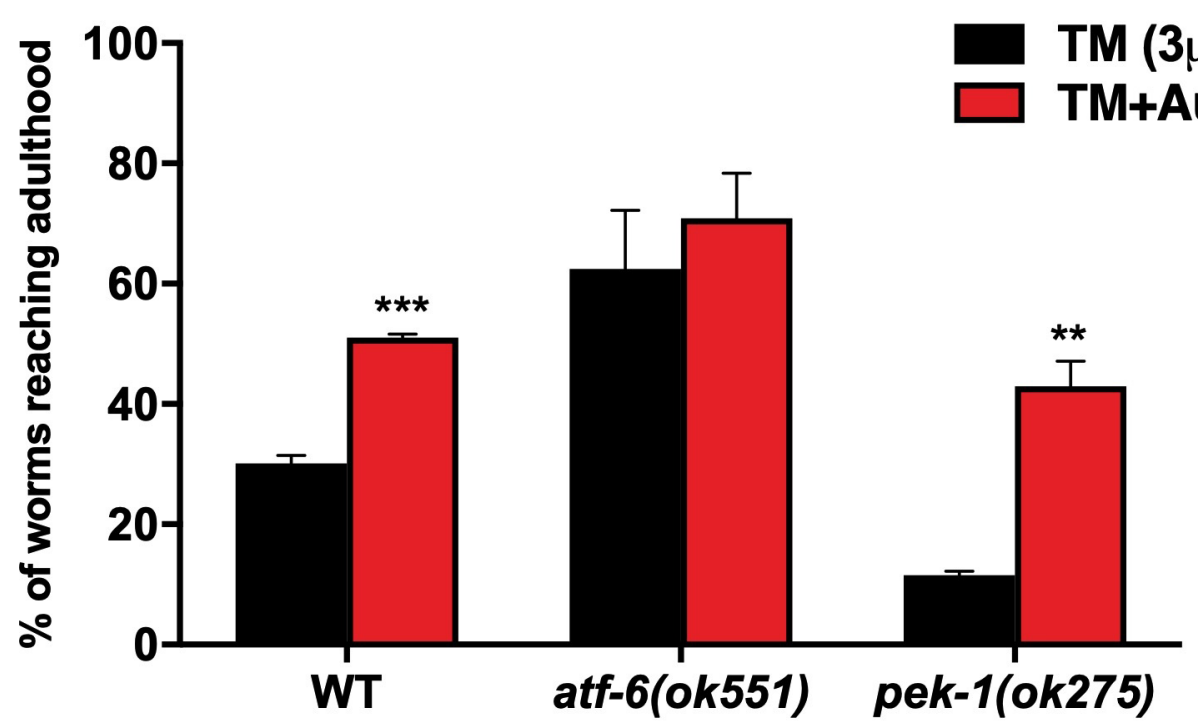

Fig. 3. The XBP-1/IRE-1 pathway of the UPR is required for auxin to induce ER stress resistance. (A) Proportion of $x b p-1(t m 2457), x b p-1(z c 12)$, and ire-1(ok799) mutants, and wild-type animals reaching the L4-adult stage on plates containing either tunicamycin $(2 \mu \mathrm{g} / \mathrm{ml})$ only, or both tunicamycin and auxin (1 mM). (B) Proportion of atf-6(ok551), pek-1(ok275), and wild-type animals reaching the L4-adult stage in the presence of tunicamycin (3 $\mu \mathrm{g} / \mathrm{ml})$ and auxin $(1 \mathrm{mM})$ or tunicamycin alone. For both $(\mathrm{A})$ and $(\mathrm{B})$ error bars show SEM from three independent experiments (multiple $t$-test with Holm-Sidak correction, ${ }^{* *} P<0.005,{ }^{* * *} P<0.0005$ auxin versus no auxin).

degradation (ERAD), ER expansion, and translational attenuation. In C. elegans, as in humans, three proteins sense ER stress and activate the UPR: the ribonuclease inositol-requiring protein-1 (IRE-1), the PERK kinase homolog PEK-1, and activating transcription factor-6 (ATF-6). Upon activation of the UPR the IRE-1/XBP-1 branch directs the majority of transcriptional regulation in response to acute ER stress, with PEK-1 and ATF-6 only playing a minor role (Shen et al., 2005).

To test whether auxin protects animals from ER stress through the IRE-1/XBP-1 pathway, we carried out ER stress survival assays on ire-1(ok799), $x b p$-(tm2457), and $x b p-1(z c 12)$ mutant animals on TM plates with or without auxin (Fig. 3A). ire-1(ok799) is a null 
mutation (Roux et al., 2016), $x b p-1\left(\right.$ tm2457) is a deletion at the $3^{\prime}$ end of the gene (Levi-Ferber et al., 2015), and $x b p-1(z c 12)$ a stop codon at the $5^{\prime}$ end of the gene (Calfon et al., 2002). Because mutants carrying these alleles are extremely sensitive to TM (Fig. 3A), (Calfon et al., 2002; Henis-Korenblit et al., 2010; Roux et al., 2016); we used a lower concentration of TM $(2 \mu \mathrm{g} / \mathrm{ml})$ in these assays, allowing us to score at least 10-20 L4/adults on TM plates. Addition of auxin improved neither developmental defects, nor survival of these mutants in the presence of TM (Fig. 3A). The stronger effect of TM on $x b p-1$ (zc12) compared to $x b p-1$ (tm2457) mutant animals suggests that $x b p-1(t m 2457)$ may be a strong hypomorph, rather than a null allele. These results show that auxin acts through the IRE-1/XBP-1 pathway to confer enhanced ER stress resistance. By contrast, further analysis showed that the putative null allele pek-1(ok275) (Henis-Korenblit et al., 2010) did not abolish the protective effect of auxin: these animals were as resistant to ER stress as wild type, ruling out a major role for the PEK-1 branch of the UPR in mediating the effect of auxin on ER stress resistance. Interestingly, the atf-6(ok551) putative null mutant (Henis-Korenblit et al., 2010) showed a strong increase in resistance to TM compared to wild-type worms, and auxin treatment only resulted in a marginal further increase (Fig. 3B). Protection against ER stress in the absence of atf- 6 has been previously described, but the mechanisms behind this effect remain elusive (Bischof et al., 2008; Burkewitz et al., 2020; Shen et al., 2005).

Together, our results point to the IRE-1/XBP-1 branch of the UPR as the main mediator of the effect of auxin on the ER stress response. These results also rule out the possibility that auxin acts by preventing the uptake of ER stress inducing drugs such as TM.

\section{DISCUSSION}

We have shown that exposure of $C$. elegans to auxin (IAA) protects animals from ER stress, both during development and in adulthood. Increased resistance to ER stress by auxin is dependent on the canonical UPR, including upstream components IRE-1/XBP-1. These results suggest that exogenous indoles can impact $C$. elegans physiology.

Indole closely resembles human and plant hormones such as serotonin and IAA, leading to the speculation that it is the archetype for cell hormones (Tomberlin et al., 2017). Our findings are consistent with recent data showing that indole and its derivatives are important signalling molecules during bacteria-nematode interactions. Caenorhabditis elegans senses and moves towards indole and indole-producing bacteria, but avoids non-indole producing pathogenic bacteria (Lee et al., 2017). Furthermore, indole-producing and non-indole-producing bacteria exert divergent effects on $C$. elegans egg-laying behaviour, and various indole derivatives also increase chemotaxis and egg-laying at low concentrations (Lee et al., 2017).

The effect of indoles and indole derivates has been also tested on nematode lifespan. Indole at a concentration range of $0.1-0.25 \mathrm{mM}$ was found to promote healthy aging (Sonowal et al., 2017) while concentrations higher than $0.5 \mathrm{mM}$ were found to reduce survival (Lee et al., 2017). By contrast, 1 mM IAA was reported to have no effect on either lifespan or mitochondrial functions (Dilberger et al., 2020; Kasimatis et al., 2018). The reasons for these different responses when using closely-related molecules are unclear. However, the beneficial effect of indole on health span was also observed in Drosophila and mice (Sonowal et al., 2017), suggesting conserved mechanisms.

The AID system is increasingly used and constantly being improved to achieve rapid and reversible protein degradation in different systems (Martinez et al., 2020; Sathyan et al., 2019). Our results suggest that caution should be applied when using this system to study stress related pathways in C. elegans, and perhaps in other species. More generally, our study illustrates how indoles and related metabolites can influence various physiological processes, with potential implications for healthy and diseased states in man (Roager and Licht, 2018).

\section{MATERIALS AND METHODS}

\section{Nematode maintenance and strains}

Animals were maintained under standard culture conditions (Brenner, 1974). The wild-type N2 (Bristol) was used as the reference strain. Strains used were obtained from the CGC and are listed as follows: ZD417 $x b p$ 1(tm2457) III; SJ17 xbp-1(zc12) III; RB925 ire-1(ok799) II; RB772 atf6(ok551) X; RB545 pek-1(ok275) X.

\section{Developmental ER stress assay}

Four to six day-1 adult worms were allowed to lay eggs on nematode growth medium plates (NGM) containing either tunicamycin only (Enzo LifeSciences; $2-3 \mu \mathrm{g} / \mathrm{ml}$ ) or tunicamycin and auxin (IAA, indole-3-acetic acid, Sigma-Aldrich; $1 \mathrm{mM}$ ), and seeded with OP50-1 bacteria. After 3-4 h we removed adult worms and counted the total number of eggs laid on each plate. L4-adult worms were scored after 4 days at $20^{\circ} \mathrm{C}$. Six plates were used for each condition. Tunicamycin and IAA stock solutions were prepared in pure DMSO and ethanol (100\%), respectively. DMSO and ethanol were used in control plates at the same percentage used in the experimental conditions $(0.03 \%$ and $0.4 \%$ for DMSO, and $0.25 \%$ for ethanol).

Statistics and significance calculations were performed by unpaired Student's $t$-test, one-way ANOVA with Tukey's multiple comparison test, or multiple $t$-test with Holm-Sidak correction, as detailed in legends. All statistical tests were carried out using GraphPad Prism 8.

\section{Adult ER stress assay}

The adult ER stress assay was performed as described previously (Taylor and Dillin, 2013). Briefly, worms were synchronized by bleaching and allowed to reach adulthood on NGM plates seeded with OP50-1. Day-1 adults were transferred on plates containing tunicamycin $(40 \mu \mathrm{g} / \mathrm{ml})$ with or without auxin $(1 \mathrm{mM})$. Survival was scored every day until all the animals were dead. Animals that crawled off the plates were not included in the analysis. $P$-values were calculated using the log-rank (Mantel-Cox) method. Statistics and significance calculations for individual lifespan studies were determined using the Oasis online software (Yang et al., 2011).

\section{Acknowledgements}

We thank members of the Palladino Lab for helpful discussions.

\section{Competing interests}

The authors declare no competing or financial interests.

\section{Author contributions}

Methodology: A.B., P.F.; Formal analysis: A.B., P.F.; Investigation: A.B., P.F.; Writing - original draft: F.P., P.F.; Writing - review \& editing: F.P., P.F.; Visualization: P.F.; Supervision: P.F.; Funding acquisition: F.P.

Funding

This work was supported by the Fondation ARC pour la recherche sur le cancer (PJA20131200036).

\section{Supplementary information}

Supplementary information available online at

https://bio.biologists.org/lookup/doi/10.1242/bio.057992.supplemental

\section{References}

Bansal, T., Alaniz, R. C., Wood, T. K. and Jayaraman, A. (2010). The bacterial signal indole increases epithelial-cell tight-junction resistance and attenuates indicators of inflammation. Proc. Natl. Acad. Sci. USA 107, 228-233. doi:10.1073/ pnas.0906112107

Bischof, L. J., Kao, C.-Y., Los, F. C. O., Gonzalez, M. R., Shen, Z., Briggs, S. P., van der Goot, F. G. and Aroian, R. V. (2008). Activation of the unfolded protein 
response is required for defenses against bacterial pore-forming toxin in vivo. PLoS Pathog. 4, e1000176. doi:10.1371/journal.ppat.1000176

Brenner, S. (1974). The genetics of Caenorhabditis elegans. Genetics 77, 71-94.

Burkewitz, K., Dutta, S., Kelley, C. A., Steinbaugh, M., Cram, E. J. and Meir, W. B. (2020). Atf-6 regulates lifespan through ER-mitochondrial calcium homeostasis. bioRxiv.

Burkewitz, K., Feng, G., Dutta, S., Kelley, C. A., Steinbaugh, M., Cram, E. J. and Mair, W. B. (2020). Atf-6 Regulates Lifespan through ER-Mitochondrial Calcium Homeostasis. Cell Rep. 32,108125. doi:10.1016/j.celrep

Calfon, M., Zeng, H., Urano, F., Till, J. H., Hubbard, S. R., Harding, H. P., Clark S. G. and Ron, D. (2002). IRE1 couples endoplasmic reticulum load to secretory capacity by processing the XBP-1 mRNA. Nature 415, 92-96. doi:10.1038/ 415092a

Camlin, N. J. and Evans, J. P. (2019). Auxin-inducible protein degradation as a novel approach for protein depletion and reverse genetic discoveries in mammalian oocytes ${ }^{\dagger}$. Biol. Reprod. 101, 704-718. doi:10.1093/biolre/ioz113

Daniel, K., Icha, J., Horenburg, C., Müller, D., Norden, C. and Mansfeld, J. (2018). Conditional control of fluorescent protein degradation by an auxindependent nanobody. Nat. Commun. 9, 3297. doi:10.1038/s41467-018-05855-5

Dilberger, B., Baumanns, S., Spieth, S. T., Wenzel, U. and Eckert, G. P. (2020) Infertility induced by auxin in PX627 Caenorhabditis elegans does not affect mitochondrial functions and aging parameters. Aging 12, 12268-12284. doi:10. 18632/aging.103413

Henis-Korenblit, S., Zhang, P., Hansen, M., McCormick, M., Lee, S.-J., Cary, M. and Kenyon, C. (2010). Insulin/IGF-1 signaling mutants reprogram ER stress response regulators to promote longevity. Proc. Natl. Acad. Sci. USA 107 9730-9735. doi:10.1073/pnas.1002575107

Hetz, C. (2012). The unfolded protein response: controlling cell fate decisions under ER stress and beyond. Nat. Rev. Mol. Cell Biol. 13, 89-102. doi:10.1038/nrm3270

Holland, A. J., Fachinetti, D., Han, J. S. and Cleveland, D. W. (2012). Inducible, reversible system for the rapid and complete degradation of proteins in mammalian cells. Proc. Natl. Acad. Sci. USA 109, E3350-E3357. doi:10.1073/ pnas.1216880109

Kasimatis, K. R., Moerdyk-Schauwecker, M. J. and Phillips, P. C. (2018). Auxinmediated sterility induction system for longevity and mating studies in Caenorhabditis elegans. G3 8, 2655-2662. doi:10.1534/g3.118.200278

Kozlowski, L., Garvis, S., Bedet, C. and Palladino, F. (2014). The Caenorhabditis elegans HP1 family protein HPL-2 maintains ER homeostasis through the UPR and hormesis. Proc. Natl. Acad. Sci. USA 111, 5956-5961. doi:10.1073/pnas. 1321698111

Lee, J.-H. and Lee, J. (2010). Indole as an intercellular signal in microbia communities. FEMS Microbiol. Rev. 34, 426-444. doi:10.1111/j.1574-6976.2009. 00204.x

Lee, J.-H., Wood, T. K. and Lee, J. (2015). Roles of indole as an interspecies and interkingdom signaling molecule. Trends Microbiol. 23, 707-718. doi:10.1016/j. tim.2015.08.001

Lee, J.-H., Kim, Y.-G., Kim, M., Kim, E., Choi, H., Kim, Y. and Lee, J. (2017) Indole-associated predator-prey interactions between the nematode Caenorhabditis elegans and bacteria. Environ. Microbiol. 19, 1776-1790. doi:10.1111/1462-2920.13649

Levi-Ferber, M., Gian, H., Dudkevich, R. and Henis-Korenblit, S. (2015) Transdifferentiation mediated tumor suppression by the endoplasmic reticulum stress sensor IRE-1 in C. elegans. Elife 4, e08005. doi:10.7554/eLife.08005

Martinez, M. A. Q., Kinney, B. A., Medwig-Kinney, T. N., Ashley, G., Ragle, J. M., Johnson, L., Aguilera, J., Hammell, C. M., Ward, J. D. and Matus, D. Q. (2020)
Rapid degradation of Caenorhabditis elegans proteins at single-cell resolution with a synthetic auxin. G3 10, 267-280. doi:10.1534/g3.119.400781

Metcalf, M. G., Higuchi-Sanabria, R., Garcia, G., Tsui, C. K. and Dillin, A. (2020) Beyond the cell factory: Homeostatic regulation of and by the UPR ${ }^{\mathrm{ER}}$. Sci. Adv. $\mathbf{6}$, eabb9614. doi:10.1126/sciadv.abb9614

Natsume, T., Kiyomitsu, T., Saga, Y. and Kanemaki, M. T. (2016). Rapid protein depletion in human cells by auxin-inducible degron tagging with short homology donors. Cell Rep. 15, 210-218. doi:10.1016/j.celrep.2016.03.001

Nishimura, K., Fukagawa, T., Takisawa, H., Kakimoto, T. and Kanemaki, M. (2009). An auxin-based degron system for the rapid depletion of proteins in nonplant cells. Nat. Methods 6, 917-922. doi:10.1038/nmeth.1401

Roager, H. M. and Licht, T. R. (2018). Microbial tryptophan catabolites in health and disease. Nat. Commun. 9, 3294. doi:10.1038/s41467-018-05470-4

Roux, A. E., Langhans, K., Huynh, W. and Kenyon, C. (2016). Reversible agerelated phenotypes induced during larval quiescence in C. elegans. Cell Metab. 23, 1113-1126. doi:10.1016/j.cmet.2016.05.024

Sathyan, K. M., McKenna, B. D., Anderson, W. D., Duarte, F. M., Core, L. and Guertin, M. J. (2019). An improved auxin-inducible degron system preserves native protein levels and enables rapid and specific protein depletion. Genes Dev. 33, 1441-1455. doi:10.1101/gad.328237.119

Shen, X., Ellis, R. E., Lee, K., Liu, C.-Y., Yang, K., Solomon, A., Yoshida, H., Morimoto, R., Kurnit, D. M., Mori, K. et al. (2001). Complementary signaling pathways regulate the unfolded protein response and are required for $C$. elegans development. Cell 107, 893-903. doi:10.1016/S0092-8674(01)00612-2

Shen, X., Ellis, R. E., Sakaki, K. and Kaufman, R. J. (2005). Genetic interactions due to constitutive and inducible gene regulation mediated by the unfolded protein response in C. elegans. PLoS Genet. 1, e37. doi:10.1371/journal.pgen.0010037 Sonowal, R., Swimm, A., Sahoo, A., Luo, L., Matsunaga, Y., Wu, Z., Bhingarde, J. A., Ejzak, E. A., Ranawade, A., Qadota, H. et al. (2017). Indoles from commensal bacteria extend healthspan. Proc. Natl. Acad. Sci. USA 114 E7506-E7515. doi:10.1073/pnas.1706464114

Taylor, R. C. and Dillin, A. (2013). XBP-1 is a cell-nonautonomous regulator of stress resistance and longevity. Cell 153, 1435-1447. doi:10.1016/j.cell.2013.05.042

Teale, W. D., Paponov, I. A. and Palme, K. (2006). Auxin in action: signalling, transport and the control of plant growth and development. Nat. Rev. Mol. Cell Biol. 7, 847-859. doi:10.1038/nrm2020

Tomberlin, J. K., Crippen, T. L., Wu, G., Griffin, A. S., Wood, T. K. and Kilner, R. M. (2017). Indole: an evolutionarily conserved influencer of behavior across kingdoms. BioEssays 39, 1600203. doi:10.1002/bies.201600203

Travers, K. J., Patil, C. K., Wodicka, L., Lockhart, D. J., Weissman, J. S. and Walter, P. (2000). Functional and genomic analyses reveal an essentia coordination between the unfolded protein response and ER-associated degradation. Cell 101, 249-258. doi:10.1016/S0092-8674(00)80835-1

Trost, M., Blattner, A. C. and Lehner, C. F. (2016). Regulated protein depletion by the auxin-inducible degradation system in Drosophila melanogaster. Fly 10 35-46. doi:10.1080/19336934.2016.1168552

Yang, J.-S., Nam, H.-J., Seo, M., Han, S. K., Choi, Y., Nam, H. G., Lee, S.-J. and Kim, S. (2011). OASIS: online application for the survival analysis of lifespan assays performed in aging research. PLoS ONE 6, e23525. doi:10.1371/journal. pone.0023525

Zhang, L., Ward, J. D., Cheng, Z. and Dernburg, A. F. (2015). The auxin-inducible degradation (AID) system enables versatile conditional protein depletion in C. elegans. Development 142, 4374-4384. doi:10.1242/dev.129635 